\title{
Artesunate has its enhancement on antibacterial activity of $\beta$-lactams via increasing the antibiotic accumulation within methicillin-resistant Staphylococcus aureus (MRSA)
}

\author{
Weiwei Jiang ${ }^{1}$, Bin $\mathrm{Li}^{1}$, Xinchuan Zheng ${ }^{2}$, Xin Liu ${ }^{2}$, Xichun Pan ${ }^{1}$, Rongxin Qing ${ }^{1}$, Yanyan Cen ${ }^{1}$, Jiang Zheng ${ }^{2}$ \\ and Hong Zhou ${ }^{1}$
}

\begin{abstract}
Methicillin-resistant Staphylococcus aureus (MRSA) has now emerged as a predominant and serious pathogen because of its resistance to a large group of antibiotics, leading to high morbidity and mortality. Therefore, to develop new agents against resistance is urgently required. Previously, artesunate (AS) was found to enhance the antibacterial effect of $\beta$-lactams against MRSA. In this study, AS was first found to increase the accumulation of antibiotics (daunorubicin and oxacillin) within MRSA by laser confocal microscopy and liquid chromatography-tandem MS method, suggesting the increased antibiotics accumulation might be related to the enhancement of AS on antibiotics. Furthermore, AS was found not to destroy the cell structure of MRSA by transmission electron microscope. AS was found to inhibit gene expressions of important efflux pumps such as NorA, NorB and NorC, but not MepA, SepA and MdeA. In conclusion, our results showed that AS was capable of enhancing the antibacterial activity of $\beta$-lactams via increasing antibiotic accumulations within MRSA through inhibiting gene expressions of efflux pumps such as NorA, NorB and NorC, but did not destroy the cell structure of MRSA. AS could be further investigated as a candidate drug for treatment of MRSA infection.
\end{abstract}

The Journal of Antibiotics (2013) 66, 339-345; doi:10.1038/ja.2013.22; published online 3 April 2013

Keywords: antibiotic; artesunate; efflux pumps; MRSA; NorA

\section{INTRODUCTION}

Since its discovery in early 1961, methicillin-resistant Staphylococcus aureus (MRSA) has now emerged as a predominant and serious pathogenic bacterium because of its resistance, leading to high morbidity and mortality. ${ }^{1,2}$ MRSA is resistant not only to $\beta$-lactams, but also to a large group of antibacterial agents such as macrolides, aminoglycosides, chloramphenicol and fluoroquinolones. ${ }^{3}$ Over the recent decades, vancomycin is the only effective antibiotic against MRSA; however, vancomycin-resistant MRSA strain has also emerged. Recently, teicoplanin, linezolid and daptomycin have been used in clinic, but the emergence of resistance will limit their application too. ${ }^{4,5}$

The major mechanism of resistance of MRSA to $\beta$-lactams is due to the acquisition of the mecA gene encoding an additional penicillinbinding protein $2 \mathrm{a}$ ( $\mathrm{PBP} 2 \mathrm{a}$ ) with lower affinity for $\beta$-lactams. ${ }^{6,7}$ In addition, decreased antibiotic accumulation and excessive $\beta$-lactamase have important roles for antibiotics resistance too. ${ }^{89}$
Decreased antibiotic accumulation may be related to increased cell wall permeability or activated efflux systems that are capable of extruding the drug or other noxious agents from the cell. ${ }^{10}$ In recent years, efflux within MRSA has attractive because of the growing data implicating efflux pumps' important contribution for MRSA resistance in many clinical strains. ${ }^{11,12}$

Efflux system can be responsible for resistance to a specific class of antibiotics or a large number of unrelated antimicrobial agents, thus conferring a multidrug-resistant phenotype. ${ }^{13}$ Several efflux pumps within MRSA have been characterized, which can be divided into five subfamilies: ATP-binding cassette superfamily, small multidrug resistance family, major facilitator superfamily, multidrug and toxic compound extrusion family, and resistance/nodulation/cell division family. Most of the pumps discovered within many MRSA clinical strains belong to major facilitator superfamily; they are NorA, NorB, NorC and so on.

\footnotetext{
${ }^{1}$ Department of Pharmacology, College of Pharmacy, The Third Military Medical University, Chongqing, China and ${ }^{2}$ Medical Research Center, Southwestern Hospital, The Third Military Medical University, Chongqing, China

Correspondence: Dr J Zheng, Medical Research Center, Southwestern Hospital, The Third Military Medical University, Gaotanyan Street 30, Shapingba District, Chongqing 400038, China.

E-mail: zhengj99219@gmail.com

Or Dr H Zhou, Department of Pharmacology, College of Pharmacy, The Third Military Medical University, Gaotanyan Street 30, Shapingba District, Chongqing 400038, China. E-mail: zhouh64@163.com
}

Received 19 July 2012; revised 20 February 2013; accepted 5 March 2013; published online 3 April 2013 
Artemisinin is an active ingredient in the Chinese herb sweet wormwood. Artemisinin and its derivates, such as artesunate (AS), dihydroartemisinin, artemether and arteether, have been clinically used to treat malaria. AS, a water-soluble hemisuccinate derivative of dihydroartemisinin, is the most widely used member of this family. Previously, in our lab AS was revealed to increase the susceptibility of various $\beta$-lactam antibiotics against Escherichia coli and MRSA. ${ }^{15,16}$

Previously, in our lab we found AS could enhance the antibiotic effect of $\beta$-lactams to MRSA via binding PBP2a and downregulating mecA gene expression. ${ }^{15}$ Furthermore, we also discovered that AS increased the daunorubicin accumulation of $S$. aureus, suggesting the enhancement of AS on antibiotics against MRSA might be related to increased antibiotics accumulation within MRSA. Therefore, herein, we further investigate the increased antibiotics accumulation within MRSA induced by AS and its possible mechanisms. Our results show that AS enhance $\beta$-lactams against MRSA via increasing antibiotics accumulations within MRSA via inhibition some important efflux pumps.

\section{RESULTS}

AS has no direct antibacterial effect, but it increases antibacterial activity of oxacillin (OXA) and ampicillin against MSRA strain WHO-2 (WHO-2)

In previous experiment, we found both MIC of ampicillin and OXA for WHO-2 were $512 \mu \mathrm{g} \mathrm{ml}^{-1}$, suggesting WHO-2 was resistant to ampicillin and OXA; MIC of AS was $>4096 \mu \mathrm{g} \mathrm{ml}^{-1}$, suggesting AS had no significant antibacterial effect in clinic because of such a high concentration. ${ }^{15}$

In present experiment, the influence of AS on the bacterial growth of WHO-2 was observed, too. The results showed AS did not influence the bacterial growth of WHO-2 with concentration from 0 to $1024 \mu \mathrm{g} \mathrm{ml}^{-1}$, only very high concentration AS $\left(2048 \mu \mathrm{g} \mathrm{ml}^{-1}\right)$ inhibited WHO-2 growth (Figure 1a). In addition, the results from bacterial dynamic growth curves also showed AS $\left(256 \mu \mathrm{g} \mathrm{ml}^{-1}\right)$ had no direct inhibition on bacterial growth, and $256 \mu \mathrm{g} \mathrm{ml}^{-1}$ of ampicillin or OXA had little effect on bacterial growth. However, there is a longer initial lag phase and an effect on the maximum growth obtained after $24 \mathrm{~h}$ of exposure, although the growth rates in logarithmic phase appear to be similar. Therefore, we considered that AS in combination with OXA or ampicillin could significantly inhibit bacterial growth from 9 to $24 \mathrm{~h}$ (Figure 1b). According to calculation formula, inhibition rate $=\left(\mathrm{OD}_{\text {antibiotics }}-\mathrm{OD}_{\text {antibiotics combined with artesunate }}\right) /$ $\left(\mathrm{OD}_{\text {antibiotics }}\right)$, at $9,12,15,18,21$ and 24 time points, the inhibition rates of AS on bacterial growth (group of OXA combined with AS) were $41.5 \%, 23.1 \%, 29.1 \%, 25.9 \%, 19.3 \%$ and $16.2 \%$, respectively; and the inhibition rates of AS on bacterial growth (group of ampicillin combined with AS) were about $62.3 \%, 29.5 \%, 25.1 \%$, $20.8 \%, 25.9 \%$ and $26.9 \%$, respectively. Above results suggested that AS had no direct antibacterial effect but could enhance antibacterial effect of ampicillin and OXA against WHO-2.

AS increases antibiotic accumulation within WHO-2 in dose- and time-dependent manners

In order to confirm the molecular mechanism of AS, the influence of AS on antibiotics accumulation was investigated using fluorospectrophotometry assay, laser confocal scanning microscope observation and liquid chromatography-tandem MS (LC-MS/MS) analysis.

Daunorubicin was an antibiotic with red autofluorescence. Therefore, it could be used as a tracer agent, and observed drug accumulation more easily and clearly. After confirmation that daunorubicin $\left(40 \mu \mathrm{g} \mathrm{ml}^{-1}\right)$ had no effect on the growth of WHO-2 (data not shown), the effect of AS on daunorubicin accumulation within WHO-2 was detected. The result from fluorescence spectrophotometer showed red fluorescence within WHO-2 increased from 0 to $20 \mathrm{~min}$ and peaked at $20 \mathrm{~min}$, displaying red fluorescence increased in dose- and time-dependent manners. After $20 \mathrm{~min}$, the fluorescence from WHO-2 treated with $>128 \mu \mathrm{g} \mathrm{ml}^{-1}$ of AS reduced; the fluorescence from WHO-2 treated with $>64 \mu \mathrm{g} \mathrm{ml}^{-1}$ of AS tended to an equilibrium value (Figure 2). The results from laser scanning microscopy showed red fluorescence within WHO-2 treated with different concentrations of AS for $20 \mathrm{~min}$ increased in a dose-dependent manner. (The higher the concentration of AS used, that stronger the fluorescence intensity of daunorubicin that existed (Figure 3).

In order to confirm whether AS directly increases OXA accumulation, OXA accumulation within WHO-2 was investigated using LC-MS/MS analysis. The results showed very low concentration of a

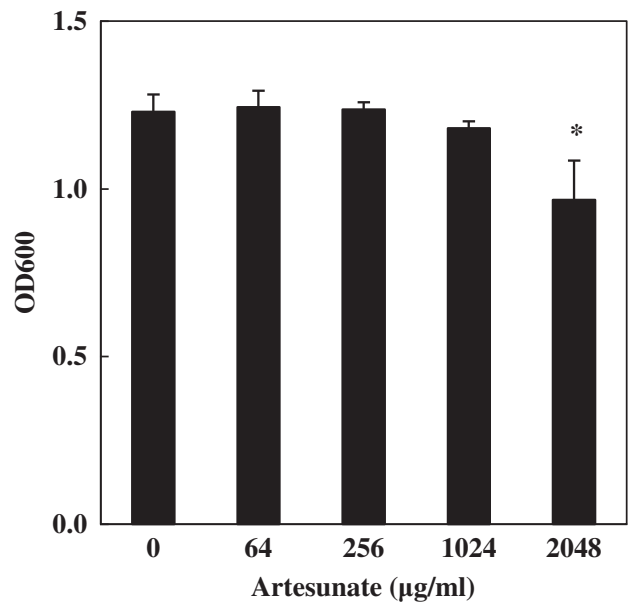

b

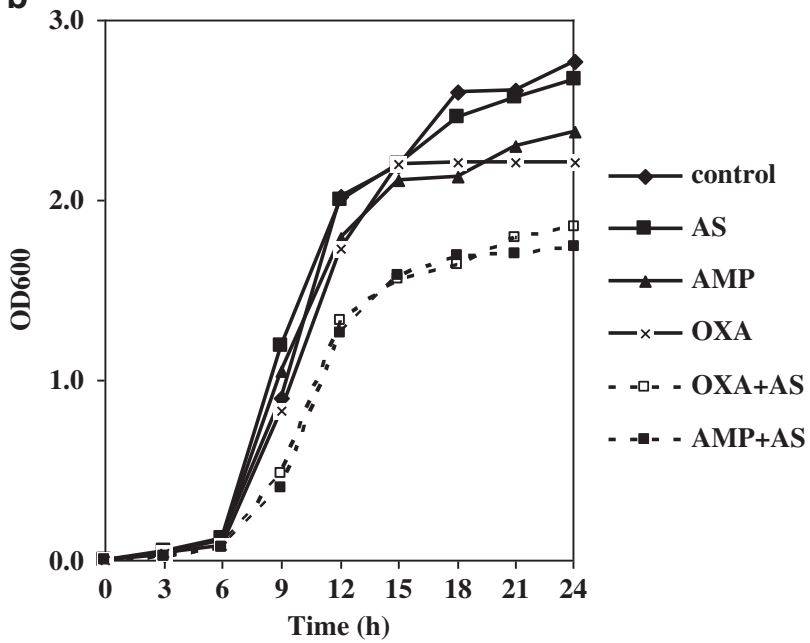

Figure 1 Artesunate (AS) increased the antibacterial activity of oxacillin (OXA) and ampicillin (AMP) against WHO-2. (a) AS had no direct antibacterial activity against WHO-2. (b) AS increased the antibacterial activity of OXA and AMP against WHO-2. The bacterial suspension was treated with nothing (control), $256 \mu \mathrm{g} \mathrm{ml}^{-1}$ of AS alone, $128 \mu \mathrm{g} \mathrm{ml}^{-1}$ of AMP or OXA alone, and $256 \mu \mathrm{gl}^{-1}$ of AS in combination with AMP or OXA (AS + AMP or AS + OXA). Then, the bacteria were cultivated for $24 \mathrm{~h}$. 
OXA was detected within the bacteria without AS treatment. However, OXA accumulation sharply increased within the bacteria treated with AS (Figure 4), confirming that AS indeed increased antibiotics accumulation.

The above three results showed that the antibacterial enhancement effect of AS was tightly related to increased antibiotics accumulation.

\section{AS does not influence the cell wall structure of WHO-2}

In order to make sure whether antibiotic accumulation was related to the destruction of the bacterial cell wall, the cell wall structure was observed by transmission electron microscope. The results showed there was no morphologic change of WHO-2 treated with different concentrations of AS (from 0 to $1024 \mathrm{gg} \mathrm{m}^{-1}$; Figure 5), suggesting antibiotic accumulation within WHO-2 was not related to cell wall destruction of WHO-2.

\section{AS reduces gene expression of important efflux pumps such as} NorA, NorB and NorC

In order to make sure that efflux pump(s) was involved in antibiotic accumulation within WHO-2, six gene expressions of efflux pumps,

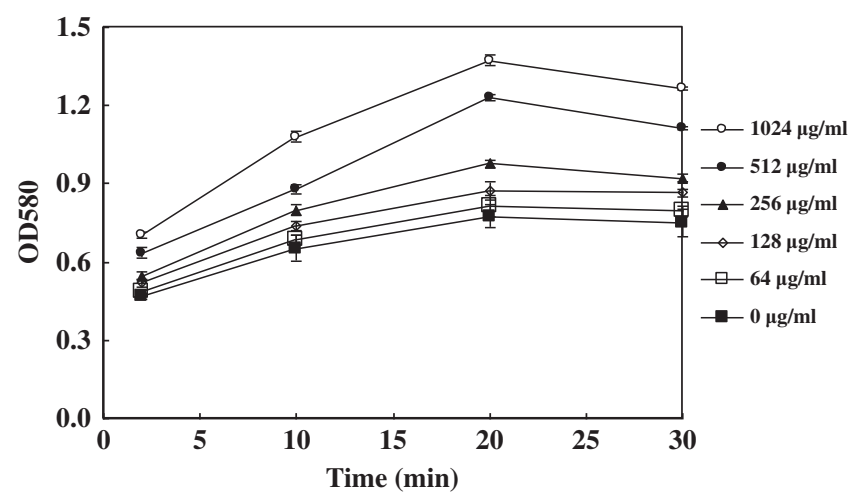

Figure 2 Artesunate (AS) increased daunorubicin accumulation within WHO-2. The fluorescence intensity of daunorubicin within the bacteria was determined by fluorescence spectrophotometer at excitation wavelength of $467 \mathrm{~nm}$ and absorption wave of $580 \mathrm{~nm}$.

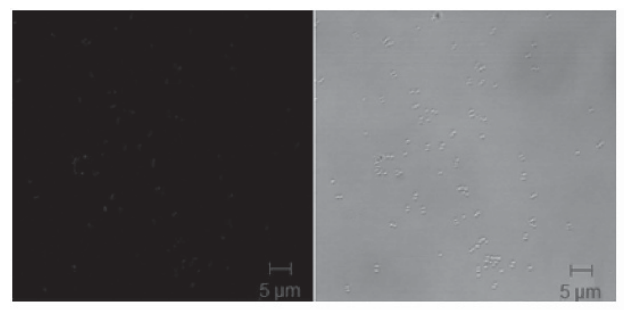

AS $0 \mu \mathrm{g} / \mathrm{ml}$

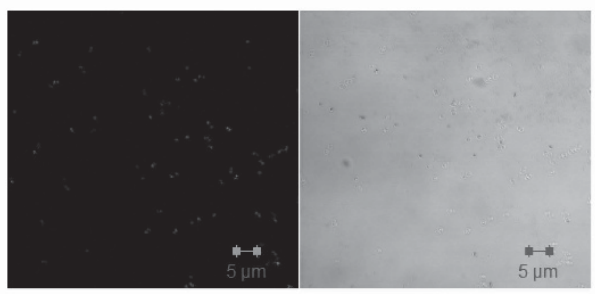

AS $256 \mu \mathrm{g} / \mathrm{ml}$
NorA, NorB, NorC, MepA, MdeA and SepA, were investigated using real-time PCR method.

The results showed there were gene expressions of NorA, NorB, NorC, MepA, MdeA and SepA within WHO-2. OXA and ampicillin upregulated all of the six gene expressions, but AS only downregulated gene expressions of NorA, NorB and NorC (Figure 6), and did not downregulate gene expressions of MepA, MdeA and SepA (data not shown). The above results suggested AS-mediated antibiotic accumulation was related to NorA, NorB and NorC not MepA, MdeA and SepA.

\section{DISCUSSION}

To the best of our knowledge, this is the first report demonstrating that AS is capable of increasing antibiotics accumulation within MRSA via inhibition of important efflux pumps, such as NorA, NorB and NorC.

As is well known, $\beta$-lactam enzymes, PBP2a and efflux pumps have very important roles during bacterial resistance. In some cases, different kinds of resistance mechanisms within same one cell result in synergistic effects. ${ }^{17}$ Within recent years, efflux pumps

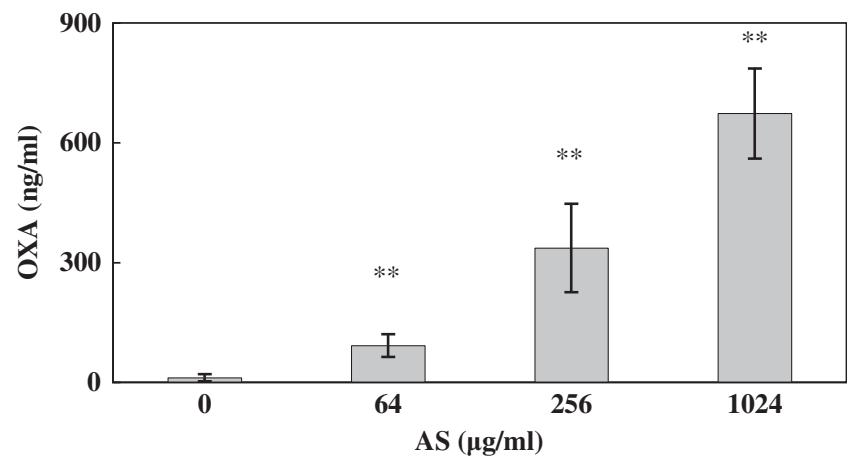

Figure 4 Artesunate (AS) increased oxacillin (OXA) accumulation within WHO-2. The filtered fluid was measured by liquid chromatography-tandem MS (LC-MS/MS) analysis using an Agilent 1100 LC system. ${ }^{* *} P<0.01$ as compared with AS $(0 \mu \mathrm{gml})$.

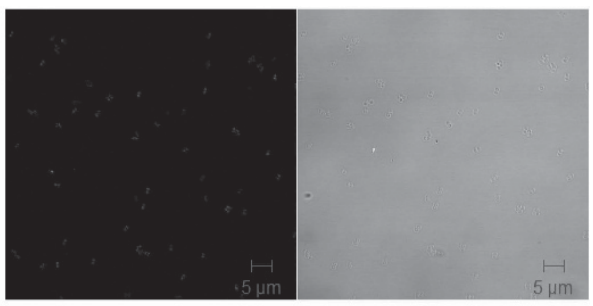

AS $64 \mu \mathrm{g} / \mathrm{ml}$

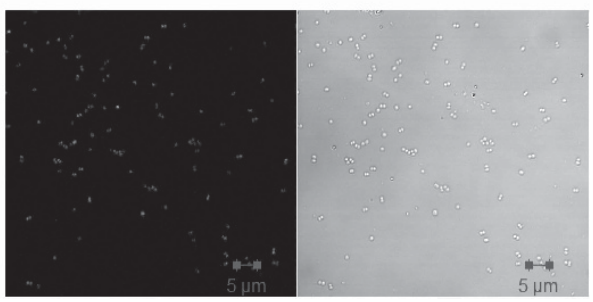

AS $1024 \mu \mathrm{g} / \mathrm{ml}$

Figure 3 Artesunate (AS) increased daunorubicin accumulation within WHO-2. The fluorescence intensity of daunorubicin in the absence and presence of AS were determined by laser scanning microscopy at excitation wavelength of $467 \mathrm{~nm}$ and absorption wave of $580 \mathrm{~nm}$. A full color version of this figure is available at The Journal of Antibiotics journal online. 


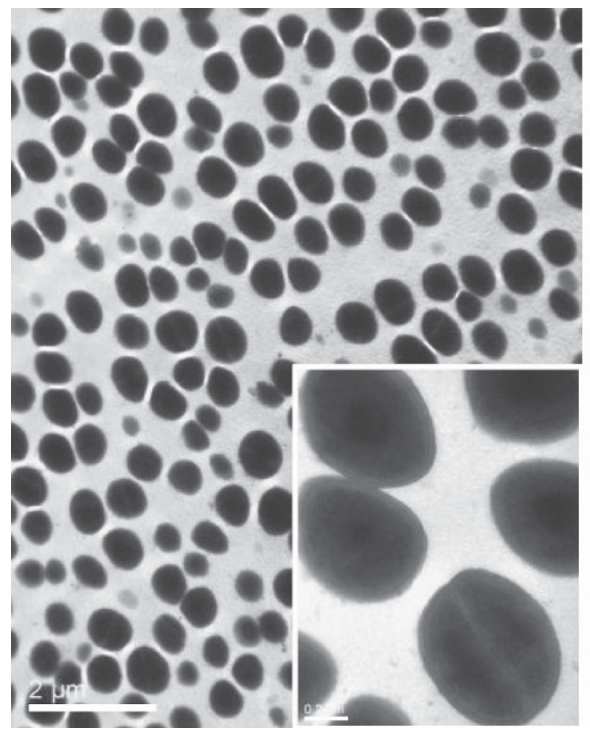

AS $0 \mu \mathrm{g} / \mathrm{ml}$

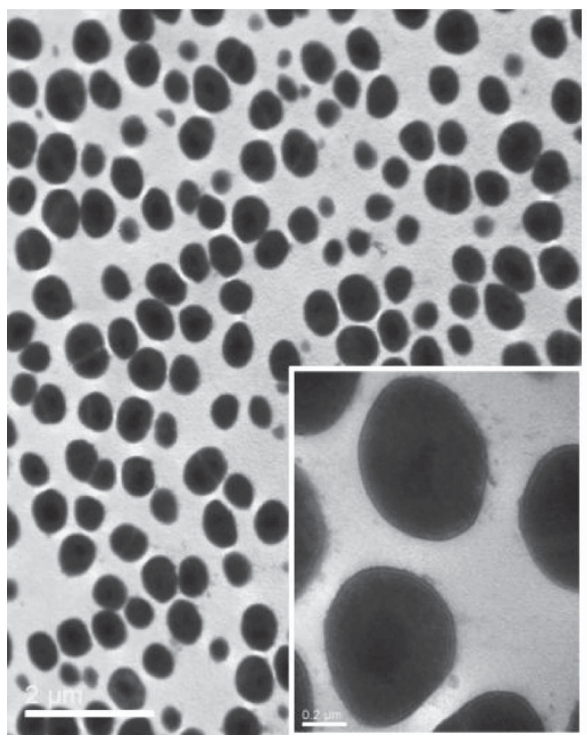

AS $256 \mu \mathrm{g} / \mathrm{ml}$

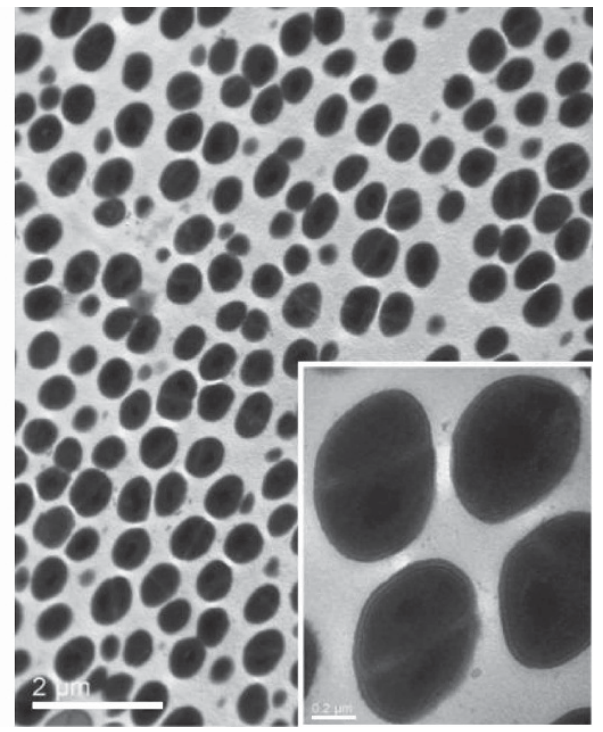

AS $64 \mu \mathrm{g} / \mathrm{ml}$

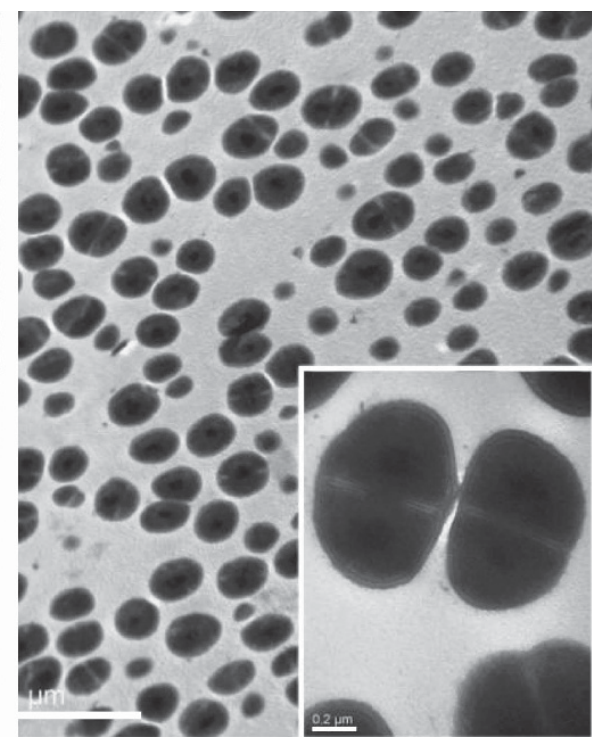

AS $1024 \mu \mathrm{g} / \mathrm{ml}$

Figure 5 Artesunate (AS) did not destroy the cell wall structure of WHO-2. The bacterial morphology was observed by transmission electron microscope.

become attractive because they make great contribution for multidrug resistant in many clinical MRSA strains. Previously, in our lab AS was revealed to increase the sensitivity of MRSA to $\beta$-lactams via binding $\mathrm{PBP} 2 \mathrm{a}$ and downregulating $m e c A$ gene expression. ${ }^{15}$ Herein, AS was further discovered to increase antibiotics accumulation within MRSA. Therefore, we considered AS affected both PBP2a and efflux pumps to enhance the antibiotic effect of $\beta$ lactams to MRSA.

Previously, in our lab AS was revealed to increase the susceptibility of various $\beta$-lactam antibiotics against $E$. coli by increasing antibiotics accumulation via inhibiting efflux pump system, AcrAB-TolC, 16 which was very important and major multidrug efflux pump system within E. coli.

To date, more than ten efflux pumps have been described within MRSA, which can be divided into five subfamilies. Most of these pumps belong to the MF superfamily, namely the chromosomally encoded NorA, NorB, NorC, MdeA and SdrM as well as the plasmid- encoded QacA/B pumps. ${ }^{18-20}$ Other types of pumps have also been discovered within MRSA such as MepA, a member of the multidrug and toxic compound extrusion family, as well as Smr, which belongs to the small multidrug resistance family, and SepA. ${ }^{11,21}$ Although these pumps show different substrate specificity, most of them are capable of extruding compounds of different chemical classes. Increased expression of efflux pumps results in decreased sensitivity for biocides, dyes and antibiotics. ${ }^{22}$ Inhibition of efflux pumps may result in increased antibiotics accumulation within the bacteria. Therefore, these pumps can be a target to solve the problem of drug resistance. Reserpine was first identified as an inhibitor of efflux pumps, but the usage was limited because of toxicity for humans. Piperine, a trans-trans isomer of 1-piperoyl-piperidine, potentiated ciprofloxacin against methicillin sensitive staphylococcus aureus and MRSA via enhancing antibiotics accumulation by inhibition bacterial efflux pumps, but their therapeutic applications needed further investigation. ${ }^{23}$ Herein, our results showed $\beta$-lactam 


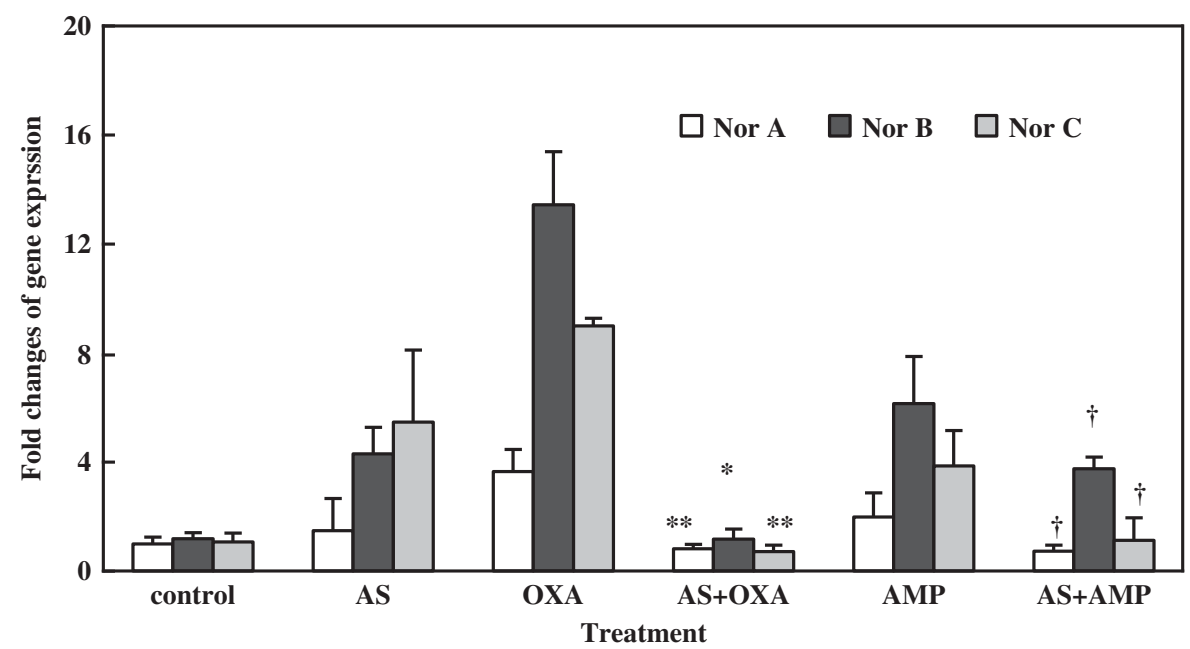

Figure 6 Artesunate (AS) reduced the gene expressions of NorA, NorB and NorC in WHO-2. The bacteria were treated as follow: broth alone (control), $256 \mu \mathrm{g} \mathrm{m} l^{-1}$ of AS alone (AS), $256 \mu \mathrm{gl}^{-1}$ of oxacillin (OXA) alone, $256 \mu \mathrm{g} \mathrm{m} l^{-1}$ of ampicillin (AMP) alone, $256 \mu \mathrm{g} \mathrm{ml}{ }^{-1}$ of OXA and $256 \mu \mathrm{g} \mathrm{ml}-1$ of AS (AS + OXA); $256 \mu \mathrm{g} \mathrm{ml}^{-1}$ of AMP and $256 \mu \mathrm{g} \mathrm{ml}^{-1}$ of AS (AS + AMP). ${ }^{* *} P<0.01$ as compared with $\mathrm{OXA},{ }^{\dagger} P<0.05$ as compared with AMP.

antibiotics could upregulate mRNA expressions of NorA, NorB, NorC, MepA, MdeA and SepA, suggesting these pumps contributed for bacterial multidrug resistant. Interestingly, AS alone also could upregulate mRNA expressions NorA, NorB and NorC because of a foreign agent for bacteria. Significantly, AS could downregulate mRNA expressions of NorA, NorB and NorC, not MepA, MdeA and SepA, suggesting AS-mediated antibiotic accumulation was related to NorA, NorB and NorC. Therefore, AS could be considered as a candidate for the treatment of infections induced by MRSA to solve the problem of drug resistance, through inhibition effect of kinds of efflux pumps. Above results were in line with previous results from our lab that AS could increase the antibiotics accumulation within E. coli via inhibition AcrB of AcrAB-TolC. ${ }^{16}$

Beta-lactams acted on the gram-positive bacterial cell wall of the bacteria through inhibition of the transpeptidase. Therefore, when the biosynthesis of the peptidoglycan is inhibited, the barrier function damaged, water came into the bacteria because of the osmotic gap between the interior and exterior of the bacteria and the bacteria simply exploded. Along with water, some water-soluble small molecules also get into the bacteria, such as $\beta$-lactams. Therefore, we considered that if there was the destruction of cell wall, the $\beta$-lactams could enter the interior of the cell of Gram-positive bacteria along with water before the bacteria exploded. The results showed there was no morphologic change of WHO-2 treated with different concentrations of AS, even when the highest concentration was $1024 \mu \mathrm{g} \mathrm{ml}^{-1}$, suggesting antibiotic accumulation within WHO-2 was not related to cell wall destruction.

In conclusion, our results showed that AS was capable of enhancing the antibacterial activity of $\beta$-lactams via increasing antibiotics accumulations within MRSA through inhibition gene expressions of efflux pumps such as NorA, NorB and NorC, but did not destroy the cell structure of MRSA. AS could be further investigated as a candidate drug for treatment of MRSA infection.

\section{MATERIALS AND METHODS}

\section{Materials}

Injectable AS was purchased from Guilin Nanyao (Guangxi, China). For in vitro and in vivo experiments, AS was dissolved in $1 \mathrm{ml}$ of $5 \%$ sodium bicarbonate (to form AS sodium), and then diluted in sterile normal saline. It was prepared freshly at the time of use. OXA was purchased from the North China Pharmaceutical Group (Shijiazhuang, China). Daunorubicin hydrochloride for injection was purchased from Pharmacia Italia SPA (Roma, Italy).

Avian myeloblastosis virus reverse transcriptase and T4 polynucleotide kinase were purchased from Promega (Madison, WI, USA). Bacterial RNAout kit was purchased from Taindz (Beijing, China). Real-time PCR Master Mix was purchased from ToYoBo (Osaka, Japan). All the primers and phosphorothioate oligonucleotides were synthesized by Invitrogen (Shanghai, China), and dissolve by aseptic deionized water.

\section{Bacterial strain and culture}

WHO-2, was kindly provided by Professor Xiaoxin Luo the Fourth Military Medical University Xi'an, China.

Single colony was picked from viable, growing Mueller-Hinton (MH) agar plate. Then, it was transferred to $5 \mathrm{ml}$ of liquid $\mathrm{MH}$ medium and cultivated aerobically at $37^{\circ} \mathrm{C}$ in orbital shaking incubator for $4 \mathrm{~h}$. The cultures were transferred to $100 \mathrm{ml}$ of fresh $\mathrm{MH}$ medium for another $12 \mathrm{~h}$. At an OD600 of $0.6-0.8$, when the bacterial culture was within the logarithmic phase of growth measured by SmartSpec 3000 spectrophotometer (Bio-Rad, Hercules, CA, USA), the suspension was centrifuged at $1500 \times g$ for $10 \mathrm{~min}$ to harvest the pellet. After washing it twice, the pellet was re-suspended by sterile normal saline. The bacteria were diluted in sterile normal saline to achieve a concentration of approximately $1.0 \times 10^{5}$ colony formation units $(\mathrm{CFU}) \mathrm{ml}^{-1}$ for experiments.

\section{Dynamic bacterial growth assay}

Different concentrations of AS $\left(0-2048 \mu \mathrm{g} \mathrm{m}^{-1}\right)$ and antibacterial agents were added to the bacterial suspension $\left(1.0 \times 10^{5} \mathrm{CFU} \mathrm{ml}^{-1}\right)$, and cultivated aerobically at $37^{\circ} \mathrm{C}$ in a slow-shaking rocking bed or a 96-well plate for $24 \mathrm{~h}$. Growth rate was determined by measuring $\mathrm{OD}_{600}$ at regular interval.

\section{Daunorubicin accumulation analysis}

Two methods (laser confocal scanning microscope and fluorospectrophotometry) were used to observe the daunorubicin accumulation within WHO-2.

In the first experiments, daunorubicin accumulation within WHO-2 was observed by fluorescence spectrophotometer (Hitachi F-2500, Tokyo, Japan). Briefly, WHO-2 was treated with different concentrations of AS in $\mathrm{MH}$ medium, ranging from 0 to $1024 \mu \mathrm{g} \mathrm{ml}^{-1}$, then the bacteria were cultured at $37^{\circ} \mathrm{C}$ in a heated, shaking environmental chamber for $12 \mathrm{~h}$, and centrifuged at 
$1500 \times g$ for $5 \mathrm{~min}$ for harvest. Bacteria were washed three times with phosphate-buffered saline (PBS) and resuspended at room temperature, OD was adjusted to 1.0 at $600 \mathrm{~nm}$. After incubation with $40 \mu \mathrm{g} \mathrm{m}^{-1}$ daunorubicin for another 5, 10, 20 and $30 \mathrm{~min}$, the bacteria were washed further with PBS for five times. Finally, daunorubicin density in WHO-2 was measured by fluorescence spectrophotometer. The emission wave length was $467 \mathrm{~nm}$ and excitation wave length was $580 \mathrm{~nm}$.

In the second experiments, daunorubicin accumulation within WHO-2 was observed under a 510 Meta confocal microscope (Zeiss, Göttingen, Germany). Briefly, WHO-2 were treated with different concentrations of AS in $\mathrm{MH}$ medium for $12 \mathrm{~h}$, and incubated with $40 \mu \mathrm{g} \mathrm{ml}^{-1}$ daunorubicin for another $20 \mathrm{~min}$. Bacteria were collected and washed five times with normal saline. Then, the bacteria were fixed on glass cover slips and observed under confocal microscope.

\section{OXA accumulation analysis}

In all, $500 \mathrm{ml}$ of WHO-2 $\left(1.0 \times 10^{5} \mathrm{CFU} \mathrm{ml}^{-1}\right)$ was treated with OXA $\left(128 \mathrm{\mu g} \mathrm{m}^{-1}\right)$ and different concentrations of AS $\left(0,64,256\right.$ and $1024 \mu \mathrm{g} \mathrm{ml}^{-}$ ${ }^{1}$ ) in $\mathrm{MH}$ medium for $3 \mathrm{~h}$. After centrifugation at $1500 \times g$ for $5 \mathrm{~min}$, the bacteria were harvested and washed three times with PBS. The bacteria was milled with liquid nitrogen and harvested with deionized water. After centrifugation by a high-speed centrifuge for $30 \mathrm{~min}$ at $13000 \times \mathrm{g}$, the supernatant was collected and mixed with methylcyanide in a volume ratio of 1:1. After another centrifugation, the supernatant was collected and mixed with carbinol in the volume ratio of 1:1. After last centrifugation, the supernatant was collected and filtered by a $0.2 \mu \mathrm{m}$ membrane filter. And then filtered fluid was measured by LC-MS/MS analysis.

LC-MS/MS analysis was performed using an Agilent 1100 LC system with a binary pump, auto sampler, column heater (kept at $30^{\circ} \mathrm{C}$ ), and degasser (Agilent Technologies, Palo Alto, CA, USA) interfaced to an API 3000 triple quadrupole mass spectrometer (Applied Biosystems, Toronto, ON, Canada). Injection volume was $5 \mu \mathrm{l}$ in the stability study to enable direct injection of analyte solutions in all tested solvents without affecting peak focusing of the early eluting analytes. An Agilent 20RBAX 300Extend-C18 column $(150 \mathrm{~mm} \times 2.1 \mathrm{~mm} ; 3.5$-micro, $300 \AA$ pore size $)$ was used for the LC separation. The flow rate of the mobile phase was $300 \mu \mathrm{min}^{-1}$. A Valco (Houston, TX, USA) divert valve was placed between the column outlet and MS source to eliminate the introduction of co-extracted matrix components into the MS instrument prior and after elution of OXA. Nitrogen was used as nebulizing and curtain gas. The entire system was controlled using the Masshunter 1.0 (Agilent Technologies, Palo Alto, CA, USA). Mobile phase A was $0.1 \%$ formic acid, and mobile phase $\mathrm{B}$ was pure methyl cyanide. The mobile phase conditions (65\% mobile phase A and 35\% mobile phase B) were used.

\section{Morphologic changes observation}

WHO-2 was treated with different concentrations of AS in $\mathrm{MH}$ medium for $12 \mathrm{~h}$, range from 0 to $1024 \mu \mathrm{g} \mathrm{ml}^{-1}$, and centrifuged at $1500 \times \mathrm{g}$ for $5 \mathrm{~min}$ for harvest. Then, the bacteria were washed three times with PBS and harvested. Morphologic changes of cell wall structure were observed by transmission electron microscope.

Briefly, the bacteria were fixed for $20 \mathrm{~min}$ in the $2.5 \%$ glutaraldehyde in $0.1 \mathrm{M}$ PBS were washed with distilled water for two times. Then, the bacteria were exposed to osmium tetraoxide for $2 \mathrm{~h}$. The dehydration of the exposed bacteria to osmium tetraoxide was dehydrated with a series of acetone $(50 \%, 70 \%$, $90 \%$, and absolute acetone), respectively, for $15 \mathrm{~min}$ each. Polymerization was done with pure epoxy result in an embedding oven at $90{ }^{\circ} \mathrm{C}$ for $2 \mathrm{~h}$ after the bacteria had been infiltrated by a mixture of acetone and epoxy resin (1:1) for $15 \mathrm{~min}$. The blocks were trimmed and cut to $60 \mathrm{~nm}$ ultra-thin sections and mounted on 200 mesh thin bar copper grids. The specimens were then stained with Reynold's stain. Each specimen was examined at $\times 10000$ and $\times 70000$ magnifications using TECNAI10 TEM (Philips, Amsterdam, Netherlands) at an accelerating voltage of $80 \mathrm{kV}$.

\section{Efflux pumps gene analysis}

The mRNA expressions of efflux pumps' genes were measured by real-time PCR. In all, $20 \mathrm{ml}$ of WHO-2 $\left(1.0 \times 10^{5} \mathrm{CFU} \mathrm{m}^{-1}\right)$ was treated with
$256 \mu \mathrm{g} \mathrm{ml}^{-1}$ OXA with or without $256 \mu \mathrm{g} \mathrm{m}^{-1}$ of AS in MH medium for $6 \mathrm{~h}$, and centrifuged at $1500 \times g$ for $5 \mathrm{~min}$ to harvest pellet. Then, the bacteria were washed three times with PBS.

Total RNA from WHO-2 was purified with Bacterial RNA out kit (Tiandz, Beijing, China) according to the manufacturer's instructions. Total RNA was reverse transcribed, RNA was diluted to $12 \mu \mathrm{l}$ and then heat denatured for $5 \mathrm{~min}$ at $70^{\circ} \mathrm{C}$. Samples were returned to ice before the addition of $15 \mu \mathrm{l}$ of master mix containing the reaction buffer, dNTPs, Taq polymerase and $2 \mu \mathrm{l}$ complementary DNA. The reverse transcribed reaction consisted of 10-min incubation at $25^{\circ} \mathrm{C}, 45$-min incubation at $42^{\circ} \mathrm{C}$, followed by a 5 -min incubation at $85^{\circ} \mathrm{C}$ termination step, and the resulting complementary DNA was stored at $-20^{\circ} \mathrm{C}$.

Real-time PCR was performed using a Real-Time PCR kit (Takara, Dalian, China), with 40 cycles of denaturation for $10 \mathrm{~s}$ at $94^{\circ} \mathrm{C}$, annealing for $10 \mathrm{~s}$ at $61{ }^{\circ} \mathrm{C}$ and extension for $30 \mathrm{~s}$ at $72^{\circ} \mathrm{C}$ by a Bio-Rad iCycler (Bio-Rad). Forward and reverse primers corresponding to genes were added to the PCR tubes and subjected to PCR amplification using primers set directly against NorA (forward: 5'-CGGTCTAGTGATA CCAGTCT- ${ }^{\prime}$, reverse: $5^{\prime}$-AACCATACCAGCACTCATAC- $3^{\prime}$ ), NorB (forward: 5'-AGCGCGTTGTCTATCTTTCC- ${ }^{\prime}$, reverse: $5^{\prime}$-GCAGGTGGTCTTGCTGAT AA- $3^{\prime}$ ), NorC (forward: $5^{\prime}$-AATGGGTTCTAAGCGACCAA-3', reverse: $5^{\prime}$-ATA CCTGAAGCAACGCCAAC- $3^{\prime}$ ), MepA (forward: $5^{\prime}$-TGCTGCTGCTCTGTTC TTTA-3', reverse: $5^{\prime}$-GCGAA GTTTCCATAATGTGC- $3^{\prime}$ ), MdeA (forward: $5^{\prime}$-GTTTATGCGATTCGAATGGTTGGT- ${ }^{\prime}$, reverse: $5^{\prime}$-ATACCTGAAGCAACG CCAAC- $3^{\prime}$ ), SepA (forward: $5^{\prime}$-GCAGTCGAGCATTTAATGGA-3' ${ }^{\prime}$, reverse: $5^{\prime}$ ACGTTGTTGCAACTGTGTAAGA-3' ${ }^{\prime}$ ) or $16 \mathrm{~S}$ (forward: $5^{\prime}$-GAGAGAAGGTG GGGATGACGT- ${ }^{\prime}$, reverse: $5^{\prime}$-AGGCCCGGGAACGTATTCAC-3'). The primers were designed by Primer Premier 5.0 (Premier Biosoft, Palo Alto, CA, USA).

To confirm amplification specificity, the PCR products were subject to a melting curve analysis, in which only one peak was observed. The amplification products were detected with a MyiQ color fluorescence real-time quantitative PCR (Bio-Rad). Three replicate reactions were performed and values were normalized to the housekeeping gene $16 \mathrm{~S}$, thus delta $\mathrm{Ct}(\mathrm{dCt})$ was calculated by subtracting the threshold cycle $(\mathrm{Ct})$ value of the endogenous control from the $\mathrm{Ct}$ value of the target gene.

\section{Statistics and presentation of data}

Each experiment was repeated a minimum of three times, each datum point represents the mean of samples. One-way analysis of variance test was used to examine the differences. A $P$-value of $<0.05$ was considered significant and a value of $<0.01$ was considered very significant; $P$-value of $>0.05$ was considered not significant.

\section{CONFLICT OF INTEREST}

The authors declare no conflict of interest.

\section{ACKNOWLEDGEMENTS}

This work was supported by a grant from the National Natural Science Foundation of China (30772618) and a grant from Innovation Foundation of the Third Military Medical University (2011XQN09). We thank Mr Xiaofeng Pan for his assistance during LC-MS/MS analysis.

1 Kopp, B. J., Nix, D. E. \& Armstrong, E. P. Clinical and economic analysis of methicillinsusceptible and -resistant Staphylococcus aureus infections. Ann. Pharmacother. $\mathbf{3 8}$ 1377-1382 (2004)

2 Shurland, S., Zhan, M., Bradham, D. D. \& Roghmann, M. C. Comparison of mortality risk associated with bacteremia due to methicillin-resistant and methicillin-susceptible Staphylococcus aureus. Infect. Control Hosp. Epidemiol. 28, 273-279 (2007).

3 Tenover, F. C. Mechanisms of antimicrobial resistance in bacteria.. Am. J. Infect. Control 34, S3-10, discussion S64-S73 (2006).

4 File, T. M. Jr. Clinical implications and treatment of multiresistant Streptococcus pneumoniae pneumonia. Clin. Microbiol. Infect. 12 (Suppl 3), 31-41 (2006).

5 Norazah, A., Salbiah, N., Nurizzat, M. \& Santhana, R. Vancomycin treatment failure in a vancomycin susceptible methicillin-resistant Staphylococcus aureus (MRSA) infected patient. Med. J. Malaysia 64, 166-167 (2009). 
6 Lim, D. \& Strynadka, N. C. Structural basis for the $\beta$-lactam resistance of PBP2a from methicillin-resistant Staphylococcus aureus. Nat. Struct. Biol 9, 870-876 (2002).

7 Martinez, M. \& Silley, P. Antimicrobial drug resistance. Handb. Exp. Pharmacol 199, 227-264 (2010).

8 Fuda, C. C. S., Fisher, J. F. \& Mobashery, S. $\beta$-Lactam resistance in Staphylococcus aureus: the adaptive resistance of plastic genome. Cell. Mol. Life Sci 62, 2617-2633 (2005).

9 Wright, G. D. The antibiotic resistome: the nexus of chemical and genetic diversity. Nat. Rev. Microbiol. 5, 175-186 (2007)

10 Nikaido, H. \& Zgurskaya, H. I. Antibiotic efflux mechanisms. Curr. Opin. Infect. Dis. 12, 529-536 (1999).

11 Longtin, J. et al. Distribution of antiseptic resistance genes qacA, qacB, and smr in methicillin-resistant Staphylococcus aureus isolated in Toronto, Canada, from 2005 to 2009. Antimicrob. Agents Chemother 55, 2999-3001 (2011).

12 Ramalhete, C. et al. Inhibition of efflux pumps in methicillin-resistant Staphylococcus aureus and Enterococcus faecalis resistant strains by triterpenoids from Momordica balsamina. Int. J. Antimicrob. Agents 37, 70-74 (2011)

13 Lomovskaya, O. \& Watkins, W. J. Efflux pumps: their role in antibacterial drug discovery. Curr. Med. Chem. 8, 1699-1711 (2001).

$14 \mathrm{Gao}, \mathrm{X}$. L. et al. A study on the active efflux system in methicillin-resistant Staphylococcus aureus. Zhong guo Wei Zhong Bing Ji Jiu Yi Xue 21, 722-725 (2009).

15 Jiang, W. et al. Artesunate in combination with oxacillin protect sepsis model mice challenged with lethal live methicillin-resistant Staphylococcus aureus
(MRSA) via its inhibition on proinflammatory cytokines release and enhancement on antibacterial activity of oxacillin. Int. Immunopharmacol 11, 1065-1073 (2011).

$16 \mathrm{Li}, \mathrm{B}$. et al. Artesunate enhances antibacterial effect of $\beta$-lactams against Escherichia coli by increasing accumulation of antibiotics via inhibiting multidrug efflux pump system AcrAB-ToIC. J. Antimicrob. Chemother 66, 769-777 (2011).

17 Sasatsu, M., Shima, K., Shibata, Y. \& Kono, M. Nucleotide sequence of a gene that encodes resistance to ethidium bromide from a transferable plasmid in Staphylococcus aureus. Nucleic Acids Res 17, 10103 (1989).

18 Ding, Y., Onodera, Y., Lee, J. C. \& Hooper, D. C. NorB, an efflux pump in Staphylococcus aureus strain MW2, contributes to bacterial fitness in abscesses. J. Bacteriol. 190, 7123-7129 (2008).

19 Gootz, T. D. The global problem of antibiotic resistance. Crit. Rev. Immunol. 30, 79-93 (2010).

20 Pantosti, A., Sanchini, A. \& Monaco, M. Mechanisms of antibiotic resistance in Staphylococcus aureus. Future Microbiol. 2, 323-334 (2007).

21 Opperman, T. J. et al. Efflux-mediated bis-indole resistance in Staphylococcus aureus reveals differential substrate specificities for MepA and MepR. Bioorg. Med. Chem 18, 2123-2130 (2010).

22 Lee, Y. S. et al. Synergistic effect of tetrandrine and ethidium bromide against methicillin-resistant Staphylococcus aureus (MRSA). J. Toxicol. Sci. 36, 645-651 (2011).

23 Khan, I. A., Mirza, Z. M., Kumar, A., Verm, V. \& Qazi, G. N. Piperine, a phytochemical potentiator of ciprofloxacin against Staphylococcus aureus. Antimicrob. Agents Chemother. 50, 810-812 (2006). 